\title{
Cost of illness of urothelial bladder cancer in Italy
}

This article was published in the following Dove Press journal:

ClinicoEconomics and Outcomes Research

24 July 2017

Number of times this article has been viewed

\author{
Claudio Gerace' \\ Francesco Montorsi ${ }^{2}$ \\ Rosa Tambaro 3 \\ Giacomo Carteni ${ }^{4}$ \\ Stefano De Luca ${ }^{5}$ \\ Marcello Tucci ${ }^{6}$ \\ Giuseppe Martorana ${ }^{7}$ \\ Umberto Basso ${ }^{8}$ \\ Leonardo Cardosi' \\ Cristina Teruzzi' \\ 'Temas s.r.l.-QuintilesIMS, Integrated \\ Market Access Department, \\ ${ }^{2}$ Department of Urology, IRCCS \\ Ospedale San Raffaele, Milano, Italy; \\ ${ }^{3}$ Department of Oncology, Istituto \\ Nazionale Tumori IRCCS Fondazione \\ Pascale, Napoli, Italy; ${ }^{4}$ A.O.R.N. \\ A. Cardarelli, Unità Operativa \\ Complessa di Oncologia Medica, \\ Napoli, Italy; ${ }^{5}$ Department of Urology, \\ Azienda Ospedaliera Universitaria San \\ Luigi Gonzaga, Orbassano, Torino Italy; \\ ${ }^{6}$ Department of Oncology, Azienda \\ Ospedaliera Universitaria San Luigi \\ Gonzaga, Orbassano, Torino Italy; \\ ${ }^{7}$ Department of Urology, Ospedale \\ Policlinico S. Orsola-Malpighi, Bologna, \\ Italy; ${ }^{8}$ Department of Clinical and \\ Experimental Oncology, Istituto \\ Oncologico Veneto IRCCS, Padova, \\ Italy
}

Correspondence: Claudio Gerace

Temas s.r.l.- QuintilesIMS, Integrated Market Access Department, Cassina Plaza, Edificio F, scala 2 Via Roma, 108, 20060 - Cassina De' Pecchi, Milano, Italy Tel +390295794452

Email claudio.gerace@quintiles.com
Background: Urothelial bladder cancer (UBC) is the ninth most common cancer worldwide. In Italy, the prevalence of the disease is approximately $10 \%$, making it the fourth most prevalent cancer in the country. The increase in prevalence requires continuous surveillance and care, resulting in a significant burden on Italian National Health Service, making any improvement to the strategy for diagnosing and treating this disease important to the medical and scientific community. The aim of this study was to evaluate the UBC cost of illness in the Italian context, collecting the total costs of the disease.

Methods: An economic analysis was carried out in the context of the National Health Service, using data collected from six centers, in order to evaluate direct costs in terms of outpatient, inpatient, and emergency care; pharmaceuticals and follow-up procedures; and indirect costs in terms of productivity losses. Data were collected through aggregated form reports, focusing on patients with an existing diagnosis of UBC who were treated in the last year. The Italian Association of Medical Oncology (AIOM) guidelines were used to identify diagnostic and therapeutic procedures. Statistical analysis was conducted to explore variations among centers. Results: The weighted mean total annual cost per patient was $€ 3,591$, where the cost for superficial disease was $€ 3,252$ and that for metastatic disease was $€ 606$. The analysis confirmed a proportional relation between disease severity and disability grade. The UBC cost of illness, considering prevalence and incidence data coming from the 2016 AIOM/Italian Association of Cancer Registries report, was $€ 1,187,036,344$. Indirect costs accounted to $44 \%$, represented by estimated productivity losses.

Conclusion: Our analysis represents the first economic study of UBC in the Italian context, as well as the first real-life evidence of the current therapeutic algorithm. This study opens the possibility for further analysis on the indirect cost components that represent a great burden for the society, especially for those in the severest stages of the disease with high disability grades. Keywords: cost of illness, cost analysis, economic evaluation, urothelial bladder cancer, direct costs, indirect costs

\section{Introduction}

Urothelial bladder cancer (UBC) is the ninth most common cancer worldwide. ${ }^{1}$ In 2016, about 26,600 new cases were expected in Italy: 21,400 among men and 5,200 among women (11\% and $3 \%$ of all incident cancers, respectively). ${ }^{2}$

UBC represents the fourth most common cancer in males, although with different percentages by age groups: $6 \%, 10 \%$, and $12 \%$ in the age groups $0-49,50-69$, and over 70 years, respectively. In women, UBC is less common and is responsible for $1 \%, 3 \%$, and $4 \%$ of all cancers in the same age groups, respectively. Trends indicate 
a statistically significant decrease in incidence among men $(-0.5 \%$ per year $)$ and a slight increase among women $(+0.1 \%$ per year), while rates of mortality have decreased among men $(-1.5 \%$ per year) and have remained constant among women. Trends appear to indicate an increase in prevalence. ${ }^{2}$

The prevalence of the disease is about $10 \%$, representing the fourth most prevalent cancer in Italy, with 253,853 cases among men and 49,695 cases among women. ${ }^{2}$

In Italy, the 5-year survival rate is about $78 \%$, which is statistically higher than the European average (68.6\%) and the average in Northern Europe $(73.0 \%) .^{2}$

More than $90 \%$ of urothelial carcinomas consist of bladder tumors that represent the primary cancer of the urinary tract, which runs from the renal pelvis to the urethra. ${ }^{3}$ Smoking and exposure to aromatic amines represent the possible risk factors. ${ }^{4} \mathrm{UBC}$ is generally not considered to be part of the family of transmission neoplasms, even though it has been reported to show a moderately higher risk in first-degree relatives. ${ }^{5}$

Early diagnosis is not predictable because of the lack of specific signs or symptoms for bladder cancer. The most common diagnostic sign is hematuria, which is frequent in urinary infections. A combined diagnostic investigation of cystoscopy with urine cytology is considered to be the best practice. $^{2}$

Clinical literature differentiates non-muscle-invasive tumors, in which the disease is confined to the surface of the inner lining of the bladder (Ta and Tis stages) or submucosa (T1 stage), from tumors with invasion of muscle layer (T2-T4 stages). ${ }^{6-8}$

According to the 2016 Italian Association of Medical Oncology (AIOM) guidelines for UBC, the therapeutic approach consists of different strategies based on the stage of the disease: transurethral resection of the bladder (TUR-B), intravesical chemotherapy or immunotherapy, cystectomy and lymphadenectomy, systemic chemotherapy, and radiotherapy.

In the presence of non-muscle-invasive neoplasia, TUR-B is considered the best treatment, with diagnostic and therapeutic intent. A second endoscopic resection (re-TUR-B), to be completed within 2-6 weeks after the first, is recommended in case of incomplete resection. About two-thirds of patients relapse within 5 years. ${ }^{9}$ Intravesical instillation of chemotherapy or immunotherapy with intravesical administration of Bacillus Calmette Guérin (BCG) can reduce this relapse rate. ${ }^{10}$

Radical cystectomy is the preferred treatment for muscleinvasive tumors, although a selected percentage of patients might be treated through bladder-sparing strategies combining radiotherapy with chemotherapy. Nevertheless, about $50 \%$ of patients may develop distant metastases, and there- fore, several trials have explored the option of neoadjuvant or adjuvant chemotherapy. Neoadjuvant chemotherapy (either with cisplatin-gemcitabine or methotrexate-vinblastine, doxorubicin and cisplatin [M-VAC] regimen) achieves a survival benefit of around $5 \%(P=0.003)$ and reduces the risk of death by $14 \% .{ }^{11-14}$ Despite favorable results, this treatment is not yet widely applied and is often replaced by a postoperative adjuvant treatment, which appears to have more toxicity and to be less effective in terms of survival benefit.

The first-choice treatment for patients with advanced UBC (metastatic disease) is platinum-based chemotherapy, with the evidence showing that cisplatin is somewhat superior to carboplatin.

Many drugs have been tested in second-line studies, but results are not entirely encouraging: low response rates, modest median survival, and relevant toxicity. Among these, a Phase III study with vinflunine versus placebo showed a modest survival advantage for vinflunine (6.9 versus 4.6 months). ${ }^{15}$

Recently, immunotherapeutic agents targeting the programmed cell death protein 1/programmed death-ligand 1 pathway (atezolizumab, nivolumab, pembrolizumab, avelumab, and durvalumab) have shown interesting and practice-changing results in several cancer types, such as non-small cell lung cancer, melanoma, urothelial cancer, and renal cancer. ${ }^{16-23}$

The management of UBC has a high impact on overall health care expenditure due to its complexity: from the high rate of recurrences to monitoring and postsurgery rehabilitation costs. All of these aspects make any evaluation an underestimate. $^{24}$

According to the 2016 AIOM-Italian Association of Cancer Registries report, the estimate of patients with UBC requiring continuous care and assistance has risen in Italy due to the increase in prevalence and reduction of mortality rate, resulting in a significant burden on the Italian health care system. Any improvements to strategies for diagnosing and treating this disease are extremely important to the medical and scientific community.

To date, no real-life data exists about the economic impact of UBC as a cost of illness analysis in Italy. The aim of this study was to evaluate the whole UBC cost of illness in the Italian context, collecting and measuring the total costs of the disease.

\section{Materials and methods Design of the study}

We planned an economic analysis across six Italian centers in order to evaluate direct and indirect costs for the care of 2,781 patients affected by UBC from the perspectives of the national health care system and the society. The main objec- 
tives of this study were to evaluate the cost of illness of this specific tumor and to collect real-life data on patient management. This analysis has been conducted on aggregated data; therefore, no single-patient level data has been collected. For this kind of analysis, IRB and authorization on the part of the Ethics Committee are not requested. In particular, under the terms of "Autorizzazione n. 9/2016, Articolo 6, Comma 2" (available in Italian language and confirming the same role as reported in "Autorizzazione n. 9/2013, Articolo 6, Comma 2" available also in English language), it is permissible to use the results of research in aggregate form for the purpose of communication and diffusion, including that which was in the scope of this publication. ${ }^{25}$

Aggregated data collection was performed with a bottom-up approach, focusing on patients with an existing diagnosis of UBC who were taken in charge in the last 12 months. ${ }^{25,26}$ In order to collect data, two different report forms were developed: one was specifically oriented to capture patient management data, and the second to collect data on resource consumption linked to each therapeutic alternative. According to the AIOM guidelines for UBC, patients were divided into three different age classes and four different disease severity stages (superficial disease, muscle-invasive disease in fit patient, muscle invasive in elderly unfit patient, metastatic disease) in report forms. ${ }^{27-29}$ Report forms were administered to clinicians (oncologists and urologists) in order to collect costs data from each center.

\section{Inclusion and exclusion criteria}

Cost analysis was based on the following inclusion criterion: patients with a diagnosis of UBC who were treated in the center in 2016.

Excluded from the analysis were patients with severe comorbidities such as cardiovascular disease and diabetes.

\section{Direct costs}

Direct cost analysis followed the gross-costing method and focused on resource consumption in terms of outpatient, inpatient, and emergency care, pharmaceuticals, and follow-up procedures. ${ }^{30}$ Physician and specialist visits, drug treatments and infusions, diagnostic examinations, and laboratory tests were based on the 2013 National Tariff Nomenclator, ${ }^{31}$ while hospitalization costs were evaluated on the basis of the national Diagnosis-Related Group (DRG) system available from the Italian Ministry of Health. ${ }^{32}$

Costs of drugs were based on the ex-factory price at net of the temporary price reductions from the 2016 Pharmaceutical Telematic Compendium and on the basis of the purchased cost related to each center. ${ }^{33}$
Costs were divided between preoperative procedures, specific treatments and postoperative tests, specialist visits, rehospitalizations, and follow-ups. We focused on the following main procedures: TUR-B, intravesical therapy, cystectomy and lymphadenectomy, chemotherapy, and radiotherapy.

\section{Indirect costs}

Indirect costs were evaluated through a human capital approach in terms of productivity losses, referring to the value of working days lost because of UBC morbidity. ${ }^{30}$ UBC-related lost working days were evaluated considering inpatient and outpatient days (visits, laboratory tests procedures), collected through the form report, and multiplied for average salary estimation, according to official inflation rates and representing a mean value for all of Italy (average earnings Bank of Italy, 2015) with the assumption that income reflects productivity. Annual lost working days were calculated excluding holidays. ${ }^{34}$

\section{Statistical analysis}

All variables were analyzed using descriptive statistics, that is, mean, standard deviation, coefficient of deviation, median, maximum, and minimum values. Results were reported as costs per patient and total costs for each expected procedure (including follow-up) and for different disease severity stages. Further statistical analyses were conducted in order to explore variations between centers.

\section{Results}

\section{Patient distribution}

Patient distribution is an important qualitative insight used to characterize UBC subjects.

Figure 1A, B shows the patient distribution based on age classes. As reported, the majority of the population is represented by subjects over 60 years old (74\%), while younger patients are fewer ( $22 \%$ for subjects $45-60$ years old and $4 \%$ for subjects under 45 years old).

Figure 1C, D shows the patient distribution based on disease severity stages and indicates that the most significant percentage of UBC patients $(62 \%)$ is in the superficial disease stage. Following disease progression, muscle-invasive percentage is $14 \%$ for cisplatinum-fit patients and $12 \%$ for cisplatinum-unfit patients. Metastatic disease represents the terminal stage and appears in $12 \%$ of subjects.

\section{Direct costs}

Figure $2 \mathrm{~A}, \mathrm{~B}$ tracks the reported costs per patient by procedures and related follow-up. All costs are reported in Euros (2016) and include pretreatments as well as follow-up inter- 
A

\begin{tabular}{lc}
\hline \multicolumn{2}{c}{ Patient distribution based on age classes } \\
\hline Age classes, years & Average \\
\hline Under 45 & $4 \%$ \\
$45-60$ & $22 \%$ \\
Over 60 & $74 \%$ \\
Total patients & $100 \%$ \\
\hline
\end{tabular}

C

\begin{tabular}{lc}
\hline \multicolumn{2}{c}{ Disease severity stage distribution } \\
\hline Disease severity stages & Average \\
\hline Superficial disease & $62 \%$ \\
Muscle-invasive in "fit patient" & $14 \%$ \\
Muscle-invasive in "elderly unfit & $12 \%$ \\
patient" & $12 \%$ \\
Metastatic disease & $\mathbf{1 0 0} \%$ \\
\hline
\end{tabular}

B

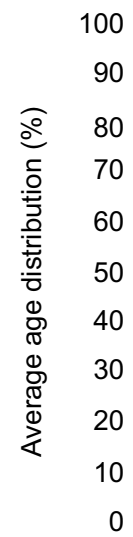

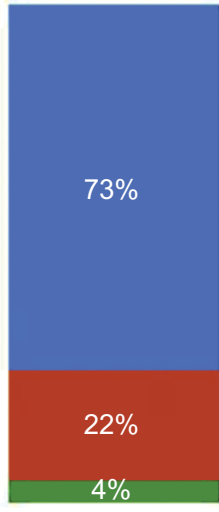

- Under $45=45-60=$ Over 60 years

D

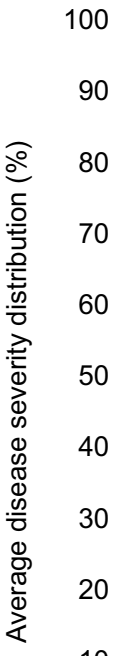

10

0

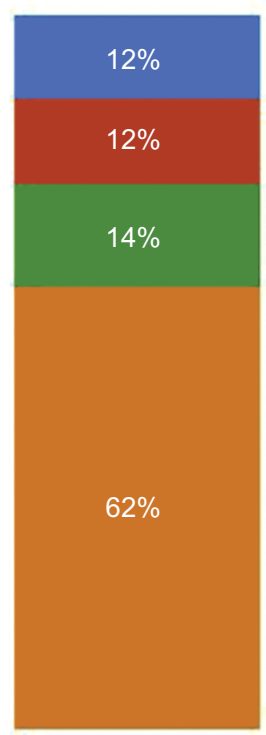

- Metastatic disease

- Muscle-invasive in "elderly unfit patient"

- Muscle-invasive in "fit patient"

= Superficial disease

Figure I Distribution based on age classes and disease severity stages.

Notes: (A and B) Patient distribution based on age classes. The majority of the population is represented by subjects over 60 years old (74\%), while younger patients are fewer ( $22 \%$ for subjects $45-60$ years old and $4 \%$ for subjects under 45 years old). (C and D) Patient distribution based on disease severity stages. The most significant percentage of urothelial bladder cancer patients (62\%) is in the superficial disease stage, muscle-invasive percentage is I4\% for cisplatinum-fit patients and I2\% for cisplatinumunfit patients. Metastatic disease represents the terminal stage and appears in $12 \%$ of subjects.

vention costs. Considering the costs for drugs, the mean of the cost per person for chemotherapy with high-cost drugs is $€ 10,147$, including first- and second-line chemotherapy. Within this therapy, the major cost item is vinflunine. In contrast, a complete scheme of chemotherapy with low-cost drugs amounts to $€ 2,438$ per person.

Cystectomy and lymphadenectomy are the most significant procedures in terms of cost per patient, at a value of $€$ $16,843$, followed by radiotherapy ( $€ 11,687)$, TUR $(€ 3,130)$,
BCG intravesical therapy ( $€ 1,341)$, and mitomycin C intravesical therapy $(€ 1,189)$.

Figure 3A, B shows the UBC direct costs stratified by disease severity stages. The weighted average of these costs, based on patient distribution by disease severity stages, highlights that cost for a patient with superficial disease is $€ 3,252$, for a muscle-invasive and fit patient $€ 2,539$, for a muscle-invasive and unfit patient $€ 1,606$, and for metastatic disease is $€ 606$. 
A

\begin{tabular}{|c|c|c|c|c|c|c|}
\hline Procedures + follow-up & Average & Median & $\begin{array}{l}\text { Standard } \\
\text { deviation }\end{array}$ & $\begin{array}{l}\text { Coefficient of } \\
\text { deviation }\end{array}$ & Minimum & Maximum \\
\hline Intravesical chemotherapy & $€ 1,189$ & $€ 977$ & $€ 591$ & $50 \%$ & $€ 460$ & $€ 2,102$ \\
\hline Intravesical therapy (BCG) & $€ 1,341$ & $€ 1,099$ & $€ 758$ & $57 \%$ & $€ 479$ & $€ 2,652$ \\
\hline $\begin{array}{l}\text { Chemotherapy (first- and second-line } \\
\text { low-cost drugs) }\end{array}$ & $€ 2,438$ & $€ 2,474$ & $€ 451$ & $19 \%$ & $€ 1,862$ & $€ 3,149$ \\
\hline TUR & $€ 3,130$ & $€ 3,061$ & $€ 230$ & $7 \%$ & $€ 2,930$ & $€ 3,487$ \\
\hline Radiotherapy & $€ 11,687$ & $€ 11,627$ & $€ 775$ & $7 \%$ & $€ 10,944$ & $€ 12,491$ \\
\hline Cystectomy and lymphadenectomy & $€ 16,843$ & $€ 16,041$ & $€ 1,914$ & $11 \%$ & $€ 15,035$ & $€ 19,921$ \\
\hline $\begin{array}{l}\text { Chemotherapy (first- and second-line } \\
\text { with high-cost drugs) }\end{array}$ & $€ 10,147$ & $€ 10,449$ & $€ 1,299$ & $13 \%$ & $€ 8,153$ & $€ 11,640$ \\
\hline
\end{tabular}

B

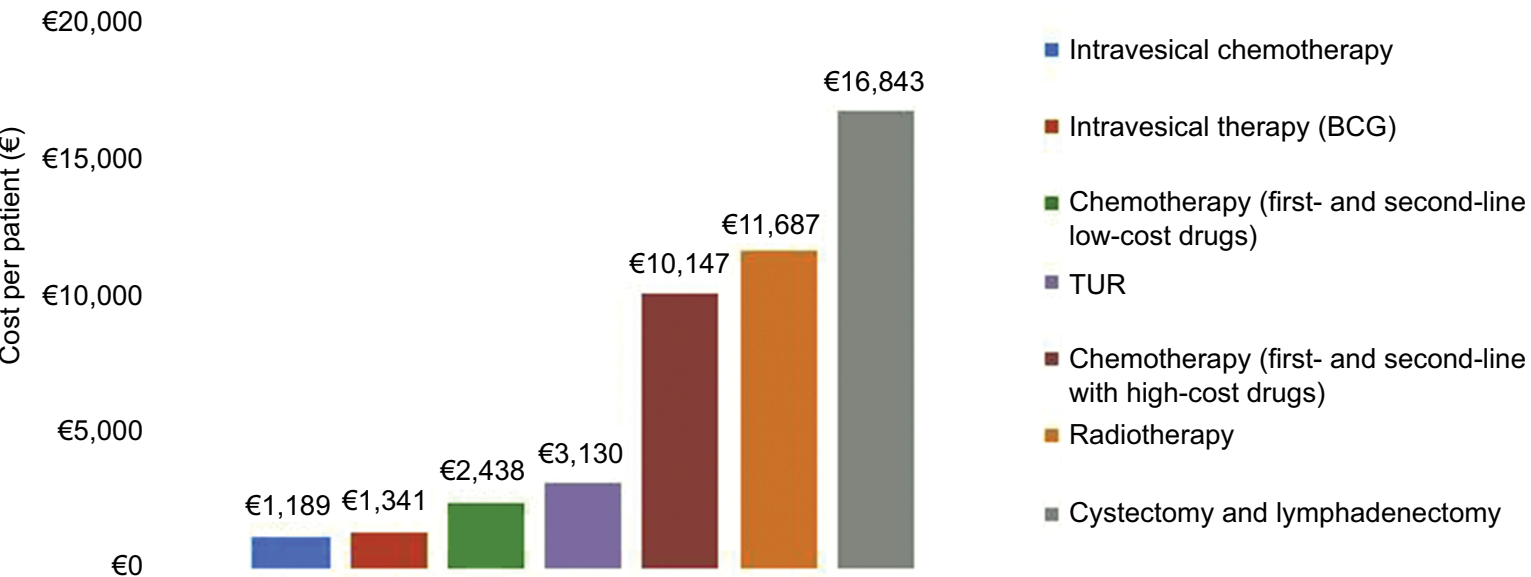

Figure 2 Cost $(€)$ per patient by procedures and follow-up.

Notes: (A and B) Reported costs per patient by procedures and related follow-up. All costs are reported in Euros (2016) and include pretreatments as well as follow-up intervention costs. Considering the costs for drugs, the mean of the cost per person for chemotherapy with high-cost drugs is $€ 10,147$, including first- and second-line chemotherapy. In contrast, a complete scheme of chemotherapy with low-cost drugs amounts to $€ 2,438$ per person. Cystectomy and lymphadenectomy are the most significant procedures in terms of cost per patient, at a value of $€ \mid 6,843$, followed by radiotherapy $(€ \mid I, 687)$, TUR (€3,I30), BCG intravesical therapy (€I,34I), and mitomycin C intravesical therapy $(€ I, 189)$.

Abbreviations: BCG, Bacillus Calmette Guérin; TUR, transurethral resection.

\section{Cost of illness}

Figure $4 \mathrm{~A}-\mathrm{C}$ shows the total costs in terms of cost of illness, including direct costs (medical examinations, hospitalizations, tests and procedures, pharmacologic therapy) and indirect costs (productivity losses). As shown in the figure, the mean cost of illness per year per patient was $€ 3,591$, with productivity losses representing $44 \%$ of the whole cost. Considering prevalence and incidence data (from 2016 AIOM registers), the total cost of UBC is $€ 1,187,036,344,56 \%$ of which is direct costs. This cost analysis was performed both from the perspectives of the National Health Service and the society.

\section{Discussion}

$\mathrm{UBC}$ has a profound impact on the society due to the severe impairment in quality of life for the affected people and the substantial economic impact on both patients and the health care system.

No significant studies on the economic burden of UBC in Italy have been carried out due to the difficulty of collecting real-life data and promoting studies for identifying the dynamics of cost of illness trends based on the evolution of the epidemiologic situation. Nevertheless, one study estimated the annual economic cost of bladder cancer across the European Union, identifying country-specific cancer costs using aggregate data on morbidity, mortality, and health care resource use. ${ }^{35}$

Our national, multicenter, retrospective analysis on the cost of illness of UBC in Italy was performed using the bottom-up approach and allowed comparisons across different cost items.

The patient distribution in our analysis shows that the majority of subjects are over $60(74 \%)$, confirming that age is a risk factor for this pathology (Figure 1A, B). Furthermore, 
A

\begin{tabular}{lcccccc}
\hline Disease severity stages & $\begin{array}{c}\text { Weighted } \\
\text { average }\end{array}$ & Median & $\begin{array}{c}\text { Standard } \\
\text { deviation }\end{array}$ & $\begin{array}{c}\text { Coefficient of } \\
\text { deviation }\end{array}$ & Minimum & Maximum \\
\hline Superficial disease & $€ 3,252$ & $€ 4,584$ & $€ 1,809$ & $34 \%$ & $€ 3,461$ & $€ 8,141$ \\
Muscle-invasive in "fit patient" & $€ 2,539$ & $€ 18,002$ & $€ 1,830$ & $40 \%$ & $€ 15,730$ & $€ 20,221$ \\
$\begin{array}{l}\text { Muscle-invasive in "elderly unfit } \\
\text { patient" }\end{array}$ & $€ 1,606$ & $€ 14,124$ & $€ 5,707$ & $43 \%$ & $€ 3,208$ & $€ 18,667$ \\
Metastatic disease & $€ 606$ & $€ 4,700$ & $€ 2,718$ & $53 \%$ & $€ 2,727$ & $€ 10,384$ \\
Total & $€ 2,001$ & $€ 8,402$ & $€ 1,148$ & $14 \%$ & $€ 6,588$ & $€ 9,478$ \\
\hline
\end{tabular}

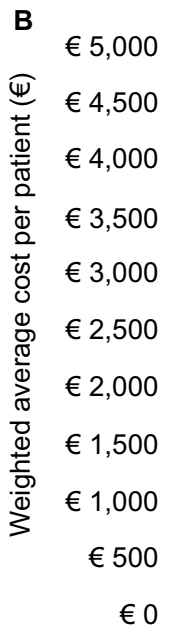

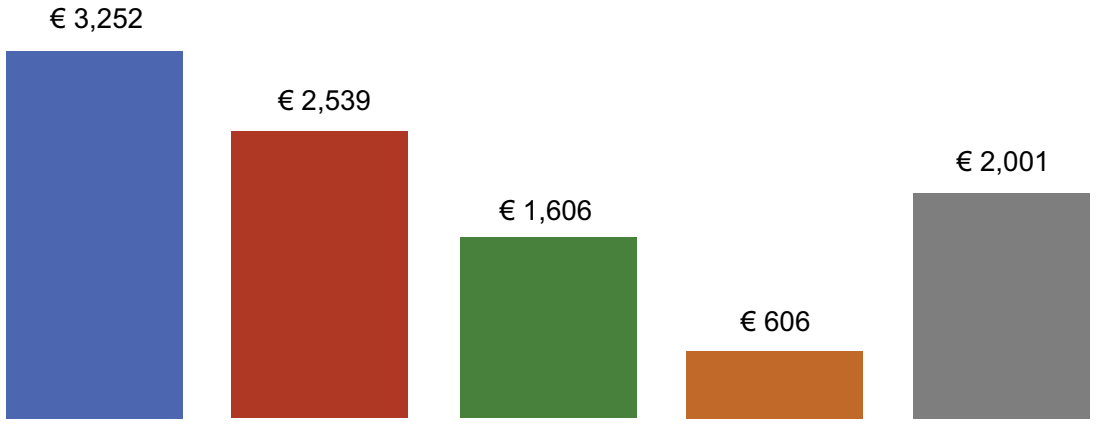

- Muscle invasive in "fit patient"

Metastatic disease

Figure 3 Cost $(€)$ per patient by disease severity stages.

Notes: (A and B) Urothelial bladder cancer direct costs stratified by disease severity stages. The weighted average of these costs, based on patient distribution by disease severity stages, highlights that cost for a patient with superficial disease is $€ 3,252$, for a muscle-invasive and fit patient $€ 2,539$, for a muscle-invasive and unfit patient $€ I, 606$, and for metastatic disease is $€ 606$.

the distribution based on disease severity stages shows that the percentage of patients with a superficial disease is higher $(62 \%)$ than the other stages, and that the percentage of affected patients decreases with a proportional relation along cancer progression. A small percentage (12\%) of patients is in the metastatic disease, associated with reduced survival and high disability grade (Figure 1C, D).

The real-life analysis of patient pathways revealed a substantial uniformity in the management of patients with UBC in the centers involved. Variability of the cost items is low and is likely related to the different settings and charging systems of individual procedures/treatments. TUR is the first treatment of choice for superficial disease, followed by BCG or mitomycin $\mathrm{C}$ intravesical therapy as adjuvant treatment. The average cost per patient for these procedures is $€ 3,130, € 1,341$, and $€$ 1,189 , respectively, including 1-year follow-up treatments.

Radical cystectomy and lymphadenectomy represent the preferred treatment strategies for muscle-invasive patients and are the first cost item with a great impact on the national health care system ( $€ 16,843)$. However, the high cost of this procedure is justified by its highly complex surgery DRG, with complications and a threshold value of 43 days. In order to improve survival probability, this radical surgery may be preceded by neoadjuvant chemotherapy or followed by adjuvant chemotherapy.

Metastatic disease is treated with M-VAC (methotrexate, vinblastine, doxorubicin and cisplatin) first-line chemotherapy for cisplatinum-fit patients and with carboplatinum/ gemcitabine for unfit patients $(€ 2,438)$. Second-line chemotherapy with vinflunine is a procedure with a profound impact in terms of cost per patient $(€ 10,147)$, as a result of the high cost of the drug. Radiotherapy ( $€ 11,687$ per patient) is a less-adopted procedure because its adoption is not considered efficacious for treating this specific tumor (Figure 2A, B).

UBC direct costs, stratified for disease severity stages, show the most important differences along metastatic disease 
A

\begin{tabular}{lcccc}
\hline \multicolumn{4}{c}{ Cost of Illness } \\
\hline Cost category & Per patient & $\begin{array}{c}\text { All patients } \\
\text { (prevalence) }\end{array}$ & $\begin{array}{c}\text { All patients } \\
\text { (incidence) }\end{array}$ & All patients (P+l) \\
\hline Direct costs & $€ 2,001$ & $€ 607,285,979$ & $€ 54,016,898$ & $€ 661,302,878$ \\
Productivity losses & $€ 1,590$ & $€ 482,790,222$ & $€ 42,943,245$ & $€ 525,733,467$ \\
Total & $€ \mathbf{3 , 5 9 1}$ & $€ \mathbf{1 , 0 9 0 , 0 7 6 , 2 0 2}$ & $€ \mathbf{9 6 , 9 6 0 , 1 4 3}$ & $€ \mathbf{1 , 1 8 7 , 0 3 6 , 3 4 4}$ \\
\hline
\end{tabular}

B

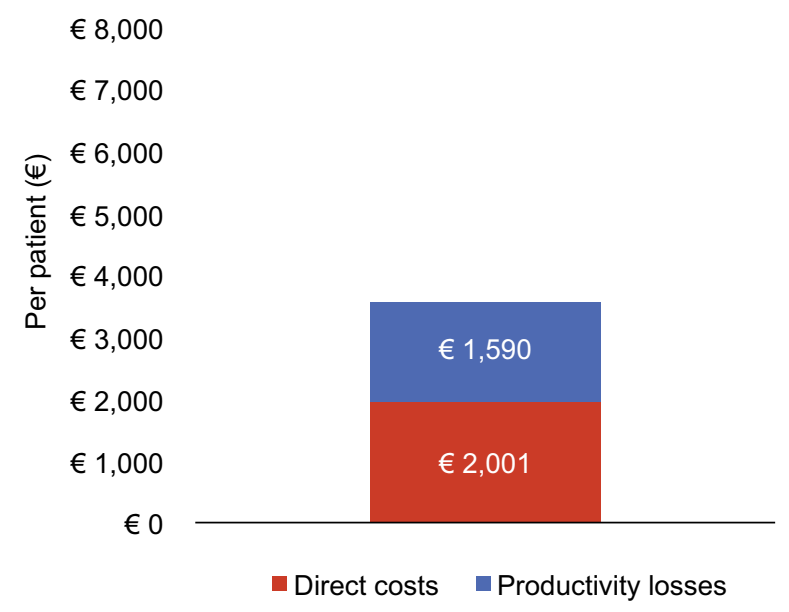

C

$€ 1,400,000,000$

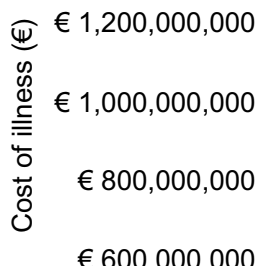

$€ 400,000,000$

$€ 200,000,000$

$€ 0$

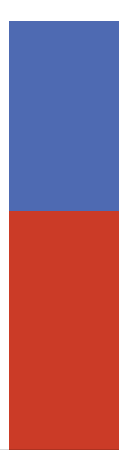

All patients $(P)$

All patients (I)

All patients $(\mathrm{P}+\mathrm{I})$

- Direct costs $n$ Productivity losses

Figure 4 Cost of illness (direct cost and productivity losses).

Notes: (A-C) Cost of illness, including direct costs (medical examinations, hospitalizations, tests and procedures, pharmacologic therapy) and indirect costs (productivity losses). The mean cost of illness per year per patient was $€ 3,59 \mathrm{I}$. Productivity losses costs are $€ I, 590$. Considering prevalence and incidence data (from 2016 Italian Association of Medical Oncology registers), the total cost of urothelial bladder cancer is $€ I, 187,036,344$. Data from AIOM report 20I6: incidence 27,000, prevalence 303,548, and incidence plus prevalence 330,548.

Abbreviations: $P$, prevalence; I, incidence.

subjects, indicating the complexity of clinical conditions and high variability in chemotherapy regimen management. In fact, second-line chemotherapy with vinflunine greatly increases per-patient costs, resulting in a high impact of this cost item.
The variability in costs related to the patient pathway and different disease severity stages is mainly due to:

- differences in the distribution of cases along the various patient pathways; 
- attractiveness of the center for certain procedures (such as urologic surgery);

- differences in the application of therapeutic and surgical protocols;

- differences in follow-up management.

Data from this study indicate that the weighted mean total cost per year for a patient with UBC was $€ 2,001$ across all disease severity stages. Furthermore, data emerging from this study highlight the need to obtain constant and thorough clinical and economic information on the number and severity of UBC cases, possibly collected in a prospective fashion.

Another important insight emerging from this analysis is the complexity of patient management levels that requires constant integrated multidisciplinary care between urologic and oncologic assets.

Our results provide descriptive data on the patterns of resource use and show that direct costs are higher than the indirect costs. Indirect costs are based on the concept of productivity losses, which can discriminate against people with no market value, such as unemployed or retired persons. In this study, we included working days lost for inpatient and outpatient visits ( 20 days per subject), in line with those reported in literature. ${ }^{35}$

Considering that direct costs data emerging from our real-life analysis amount to $€ 661,302,878$, the Italian cost of illness is similar to the data reported by Leal et al. ${ }^{35}$ If one also considers productivity losses, the total cost of disease becomes $€ 1,187,036,344$, suggesting the profound burden that this pathology places on the society.

According to our analysis, the total annual UBC costs account for $1.08 \%$ of Italian public health care spending for 2016 ( $€ 110.3$ billion). Furthermore, direct costs per patient $(€ 2,001)$ represent $10 \%$ of the overall per capita health care expenditure of all cancers $(€ 114){ }^{36}$

There are some limitations to this study, and the results are likely to underestimate total disease costs for various reasons. First of all, direct health care costs did not include general practitioner visits and out-of-pocket expenditures. Furthermore, direct non-health care costs were not included in the analysis.

Another possible bias concerns the choice of the centers in which the analysis was conducted. Four of them are located in the northern area and two in the southern area. This selection may not be representative of the whole territorial distribution of the affected population in Italy. However, centers were chosen on the basis of excellence of their operational protocols.

Moreover, interregional mobility for cancer is a usual phenomenon in Italy because of the major attractiveness of excellent centers, such as those selected for this analysis. Another limitation of analysis is the use of national tariffs and DRG for evaluating direct costs related to the health resources consumption, although this approach is widely used as a good proxy of the real costs. ${ }^{37-40}$

For the indirect cost estimation, productivity losses were based on the hypothesis of 220 working days as a good proxy of real life. ${ }^{34}$ The costs of productivity losses could be overestimated because of the high percentage of the elderly or otherwise nonproductive population represented in this study. Informal care costs were not included in this analysis.

Our analysis represents the first study of the economic cost of illness of UBC in the Italian context, as well as the first real-life evidence of the current therapeutic algorithm. This study opens the possibility for further analysis of the indirect cost components that represent a great burden for society, especially concerning the stages of the disease associated with a high disability grade, during which informal care is necessary and the impact on quality of life is greater.

Additional studies are required to address the evidence gaps concerning the effectiveness and cost-effectiveness of existing treatment strategies, and to evaluate surveillance technologies for UBC that can inform optimal resource allocation for the management of the disease.

\section{Acknowledgments}

This analysis was financially supported by an unconditional grant from Roche S.p.a.

The authors would like to thank Professor Giorgio Colombo of University of Pavia, Department of Drug Sciences, School of Pharmacy (Pavia) for the critical review of the manuscript; Giuseppe Di Ponzio for data acquisition; and Istituto Nazionale Tumori di Napoli IRCCS "Fondazione G. Pascale", Policlinico Sant'Orsola Malpighi (Bologna), Azienda Ospedaliera di Rilievo Nazionale Antonio Cardarelli (Napoli), Azienda Ospedaliera Universitaria San Luigi Gonzaga (Orbassano), IRCCS Ospedale San Raffaele (Milano), IRCCS Istituto Oncologico Veneto (Padova) for support in collecting data.

\section{Disclosure}

The authors report no conflicts of interest in this work.

\section{References}

1. AIOM Guidelines, 2015. Available from: http://www.aiom.it/professionisti/documenti-scientifici/linee-guida/vescica/1,1030,1,2. Accessed September 15, 2015.

2. I numero del cancro in Italia; 2016 [The number of cancer in Italy; 2016]. Available from: http://www.registri-tumori.it/cms/it/node/4572. Accessed September 27, 2016. 
3. Eble JN, Sauter G, Epstein JI, Sesterhenn IA, editors. World Health Organization classification of tumors. Pathology and genetics of tumors of urinary system and male genital organs. Lyon: IARC Press; 2004.

4. Negri E, La Vecchia C. Epidemiology and prevention of bladder cancer. Eur J Cancer Prev 2001;10(1):7-14.

5. Aben KK, Witjes JA, Schoenberg MP, et al. Familial aggregation of urothelial cell carcinoma. Int J Cancer. 2002;98(2):274-278.

6. Sobin DH, Wittekind Ch, editors. In: TNM Classification of Malignant Tumors. 6th ed. New York, NY: Wiley-Liss; 2002:199-202.

7. Sauter G, Algaba F, Amin M, et al. Tumors of the urinary system: non-invasive urothelial neoplasias. In: Eble JN, Sauter G, Epstein J1, Sesterhenn I, editors. WHO Classification of Tumors of the Urinary System and Male Genital Organs. Lyon: IARCC Press; 2004:29-34.

8. Lopez-Beltran A, Montironi R. Non-invasive urothelial neoplasms: according to the most recent WHO classification. Eur Urol. 2004;46(2):170-176.

9. Oosterlink W, Lobel B, Jaske G, Malmström PU, Stöckle M, Sternberg CN. Guidelines on bladder cancer. Eur Urol. 2002;41:105-112.

10. Sylvester RJ, van der Meijden AP, Oosterlinck W, et al. Predicting recurrence and progression in individual patients with stage TaT1 bladder cancer using EORTC risk tables: a combined analysis of 2596 patients from seven EORTC trials. Eur Urol. 2006;49(3):466- 477.

11. Galsky MD, Pal SK, Chowdhury S, et al. Comparative effectiveness of gemcitabine plus cisplatin versus methotrexate, vinblastine, doxorubicin, plus cisplatin as neoadjuvant therapy for muscle-invasive bladder cancer. Cancer. 2015;121(15):2586-2593.

12. Advanced Bladder Cancer (ABC) Meta-analysis Collaboration. Neoadjuvant chemotherapy in invasive bladder cancer: a systematic review and meta-analysis. Lancet. 2003;361:1927-1934.

13. Advanced Bladder Cancer (ABC) Meta-analysis Collaboration. Neoadjuvant chemotherapy in invasive bladder cancer: update of a systematic review and meta-analysis of individual patient data advanced bladder cancer (ABC) meta-analysis collaboration. Eur Urol. 2005;48(2):202-205.

14. Advanced Bladder Cancer (ABC) Meta-analysis Collaboration. Adjuvant chemotherapy in invasive bladder cancer: a systematic review and meta-analysis of individual patient data. Eur Urol. 2005;48(2):189-199.

15. Bellmunt J, Theodore C, Demkov T, et al. Randomized phase III trial of vinflunine (V) plus best supportive care(B) compared with $B$ alone a after a platinum-containing regimen in patients with advanced transitional cell carcinoma of the urothelial tract. J Clin Oncol 2009;27(27):4454-4461.

16. Powles T, Eder JP, Fine GD, et al. MPDL3280A (anti-PD-L1) treatment leads to clinical activity in metastatic bladder cancer. Nature. 2014;515(7528):558-562.

17. Rosenberg JE, Hoffman-Censits J, Powles T, et al. Atezolizumab in patients with locally advanced and metastatic urothelial carcinoma who have progressed following treatment with platinum-based chemotherapy: a single-arm, multicentre, phase 2 trial. Lancet. 2016;387(10031):1909-1920.

18. Sharma P, Bono P, Kim JW, et al. Efficacy and safety of nivolumab monotherapy in metastatic urothelial cancer (mUC): Results from the phase I/II CheckMate 032 study. J Clin Oncol. 2016;34(15 Suppl): 4501.

19. Balar AV, Galsky MD, Loriot Y, et al. Atezolizumab (atezo) as firstline (1L) therapy in cisplatin-ineligible locally advanced/metastatic urothelial carcinoma (mUC): Primary analysis of IMvigor210 cohort 1. J Clin Oncol. 2016;34 (Suppl; Abstr LBA4500)

20. Dreicer R, Hoffman-Censits J, Flaig TW, et al. Updated efficacy and >1-year follow up from IMvigor 210: Atezolizumab (atezo) in platinum (plat) treated locally advanced/metastatic urothelial carcinoma (mUC). J Clin Oncol. 2016;34(Suppl; Abstr 4515)
21. Massard C, Gordon MS, Sharma S, et al. Safety and efficacy of durvalumab (MEDI4736), a PD-L1 antibody, in urothelial bladder cancer. $J$ Clin Oncol. 2016;34(26):3119-3125.

22. Apolo AB, Infante JR, Hamid O, et al. Avelumab (MSB0010718C; anti-PD-L1) in patients with metastatic urothelial carcinoma from the JAVELIN solid tumor phase $1 \mathrm{~b}$ trial: analysis of safety, clinical activity, and PDL1 expression. J Clin Oncol. 2016;34(Suppl; Abstr 4514)

23. Plimack ER, Bellmunt J, Gupta S, et al. Pembrolizumab (MK-3475) for advanced urothelial cancer: updated results and biomarker analysis from KEYNOTE-012. J Clin Oncol. 2015;33(Suppl 15):4502.

24. Racioppi M, Volpe A, Falabella R, et al. The cost of treatment and followup of bladder cancer in Italy. Arch Ital Urol Androl. 2007;79(3):111-117.

25. Authorization no. 9/2013 - General Authorization to Process Personal Data for Scientific Research Purposes. Available from: http://www. garanteprivacy.it/web/guest/home/docweb/-/docweb-display/docweb/3039584. Accessed December 12, 2013.

26. Schinco P, Cultrera D, Valeri F, et al. Cost-consequence analysis of long-term prophylaxis in the treatment of von Willebrand disease in the Italian context. Clinicoecon Outcomes Res. 2014;7:17-25

27. Rosenberg JE, Carroll PR, Small EJ. Update on chemotherapy for advanced bladder cancer. $J$ Urol. 2005;174(1):14-20.

28. Bellmunt J, Petrylak DP. New therapeutic challenges in advanced bladder cancer. Semin Oncol. 2012;12(5):598-607.

29. Galsky MD, Hahn NM, Rosenberg J, et al. A consensus definition of patients with metastatic urothelial carcinoma who are unfit for cisplatinbased chemotherapy. Lancet Oncol. 2011;12(3):211-214.

30. Tarricone R. Cost-of-illness analysis. What room in health economics? Health Policy. 2006;77(1):51-63.

31. Health Ministry, 2013 National Tariff Nomenclator. Nomenclatore delle Prestazioni di assistenza specialistica ambulatoriale- Ministero della Salute, 2013. Italian. Available from: http://www.salute.gov.it/portale/ temi/p2_6.jsp?id=3662\&area=programmazioneSanitariaLea\&menu= vuoto.

32. Health Ministry, 2013 DRG Tariff- TUC Tariffa Unica Convenzionale, Conferenza delle Regioni e delle Province Autonome, compensazione interregionale della mobilità sanitaria Testo Unico.Versione in vigore per le attività dell'anno 2016. Rome, February 02, 2017. Italian. Available from: http://www.salute.gov.it/portale/temi/p2_6.jsp?id=3662\&ar ea=programmazioneSanitariaLea\&menu=vuoto.

33. Pharmaceutical Telematic Compendium 2016, Banca dati del farmaco e parafarmaco - Farmadati. Available from: http://www.farmadati.it/.

34. Marcellusi A, Viti R, Capone A, Mennini FS. The economic burden of $\mathrm{HCV}$-induced diseases in Italy. A probabilistic cost of illness model. Eur Rev Med Pharmacol Sci. 2015;19(9):1610-1620.

35. Leal J, Luengo-Fernandez R, Sullivan R, Witjes JA. Economic Burden of Bladder Cancer across the European Union. Eur Urol. 2016;69(3):438-447.

36. 8th Report on the patient's condition in oncology, Rome May 12-15, 2016, XI National Day of the Oncologist. Available from: https://www. favo.it/phocadownload/Ottavo_Rapporto_FAVO_2016.pdf.

37. Benedini V, Caporaso N, Corazza GR, et al. Burden of Crohn's disease: economics and quality of life aspects in Italy. Clinicoecon Outcomes Res. 2012;4:209-218.

38. Colombo G, Altomare G, Peris K, et al. Moderate and severe plaque psoriasis: cost-of-illness study in Italy. Ther Clin Risk Manag. 2008;4(2):559-568.

39. Colombo GL, Di Matteo S, Vinci M, et al. A cost-of-illness study of spina bifida in Italy. Clinicoecon Outcomes Res. 2013;2(5):309-316.

40. Fattore G1, Torbica A. Inpatient reimbursement system in Italy: how do tariffs relate to costs? Health Care Manag Sci. 2006;9(3): $251-258$. 


\section{Publish your work in this journal}

ClinicoEconomics and Outcomes Research is an international, peerreviewed open-access journal focusing on health technology assessment, pharmacoeconomics and outcomes research in the areas of diagnosis, medical devices, and clinical, surgical and pharmacological intervention. The economic impact of health policy and health systems organization also constitute important areas of coverage. The manuscript management system is completely online and includes a very quick and fair peer-review system, which is all easy to use. Visit http://www.dovepress.com/testimonials.php to read real quotes from published authors.

Submit your manuscript here: https://www.dovepress.com/clinicoeconomics-and-outcomes-research-journal 